\title{
Direct measurement of product of the electron mobility and mean free drift time of CdZnTe semiconductors using position sensitive single polarity charge sensing detectors
}

\author{
Z. He, ${ }^{\text {a) }}$ G. F. Knoll, and D. K. Wehe \\ Department of Nuclear Engineering and Radiological Sciences, University of Michigan, Ann Arbor, \\ Michigan 48109
}

(Received 18 December 1997; accepted for publication 12 August 1998)

\begin{abstract}
This article describes novel techniques to directly measure the electron mobility and mean free drift time product $\mu_{e} \tau_{e}$ in semiconductor detectors. These methods are based on newly developed single polarity charge sensing and depth sensing techniques. Compared with conventional methods based on the Hecht relation, the new methods do not involve curve fitting, are less sensitive to the variation of pulse rise times, and allow the use of higher energy $\gamma$ rays typical of many applications. (C) 1998 American Institute of Physics. [S0021-8979(98)01122-0]
\end{abstract}

\section{INTRODUCTION}

The transport properties of charge carriers are important intrinsic parameters of semiconductor materials, and are used in evaluating their potential for various applications. In particular, the single polarity charge sensing technique has been recently proven effective for wide band-gap semiconductor $\gamma$-ray detectors. ${ }^{1}$ For this technique, the electron $\mu_{e} \tau_{e}$ product is one of the key parameters that determines the performance of such a device using electron charge sensing.

The conventional methods ${ }^{2}$ of measuring the product of mobility $\mu_{e}$ and mean free drift time $\tau_{e}$ of electrons in a planar detector is based on the Hecht relation: ${ }^{3}$

$$
Q=N_{0} e_{0} \frac{\lambda_{e}}{D}\left[1-\exp \left(-\frac{D}{\lambda_{e}}\right)\right],
$$

where $Q$ is the induced charge on one of the planar electrodes (cathode or anode), $N_{0}$ is the number of electron-hole pairs generated by the $\gamma$ ray, $e_{0}$ is the charge of an electron, $\lambda_{e}$ is the mean free drift length of an electron, and $D$ is the thickness of the semiconductor. $\lambda_{e}$ is defined as:

$$
\lambda_{e}=\mu_{e} \tau_{e} E,
$$

where $E$ is the electric field intensity $E=V / D$ (for planar electrode configurations) and $V$ is the bias voltage between the cathode and the anode.

In conventional measurements of $\mu_{e} \tau_{e}$, the electronhole pairs need to be generated near the cathode surface so that the induced signal on one of the electrodes is from the drift of electrons only. This can be implemented using a laser pulse, $\alpha$ particles, or low energy x rays incident from the cathode surface. By measuring the variation of photopeak amplitudes, assumed to be proportional to the total induced charge $Q$ of Eq. (1), as a function of bias voltage, the $\mu_{e} \tau_{e}$ value can be estimated from a curve fitting procedure using Eqs. (1) and (2).

There are several factors that may affect the precision of these measurements. For example, consider the assumption

\footnotetext{
${ }^{a}$ Electronic mail: hezhong@engin.umich.edu
}

that the measured pulse amplitudes are proportional to the total induced charge $Q$ at different bias voltages. This is only true when the shaping time constant is much longer than the pulse rise time, which is the charge drift time across the detector thickness. In practice, the pulse rise time can be comparable to the shaping time, and the variation of ballistic deficit due to changes in pulse rise time cannot be ignored. For instance, the drift time of electrons across $1 \mathrm{~cm}$ of $\mathrm{CdZnTe}$ is $\sim 1 \mu$ s at $1 \mathrm{kV}$ bias voltage and is longer at lower voltages. A $10 \mu$ s shaping time constant may cause significant ballistic deficit, especially at low bias voltages. The correction for this systematic error is not trivial. One could use a thin detector to reduce the ballistic deficit, but the electron trapping becomes small which increases the measurement error in the $\mu_{e} \tau_{e}$ value. Second, a curve fitting procedure must be employed to give an estimate of $\mu_{e} \tau_{e}$, which complicates the estimation of measurement precision. Third, the charge trapping conditions near surfaces may be significantly different from that within the bulk. If electron-hole pairs are generated too close to the surface, the measurement results may be different from those obtained if the electron-hole pairs are generated within the bulk of the semiconductor. Finally, the measurements based on the Hecht relation rely on the assumption that the charge trapping is uniform within the bulk. This assumption may not be valid due to the nonuniformity of the material.

This article describes different approaches in which $\mu_{e} \tau_{e}$ can be directly measured simply. These methods are based on the newly developed single polarity charge sensing technique $^{1}$ and the depth sensing method. ${ }^{4,5}$ These new methods have the potential to reduce all the systematic errors mentioned above.

\section{DIRECT MEASUREMENT OF $\mu_{e} \tau_{e}$ BY MEASURING PHOTOPEAK AMPLITUDES VERSUS DRIFT LENGTH $z$}

If the fractional electron loss $(d N / N)$ per unit path length in a semiconductor can be considered as a constant $1 / \lambda_{e}$, i.e., 


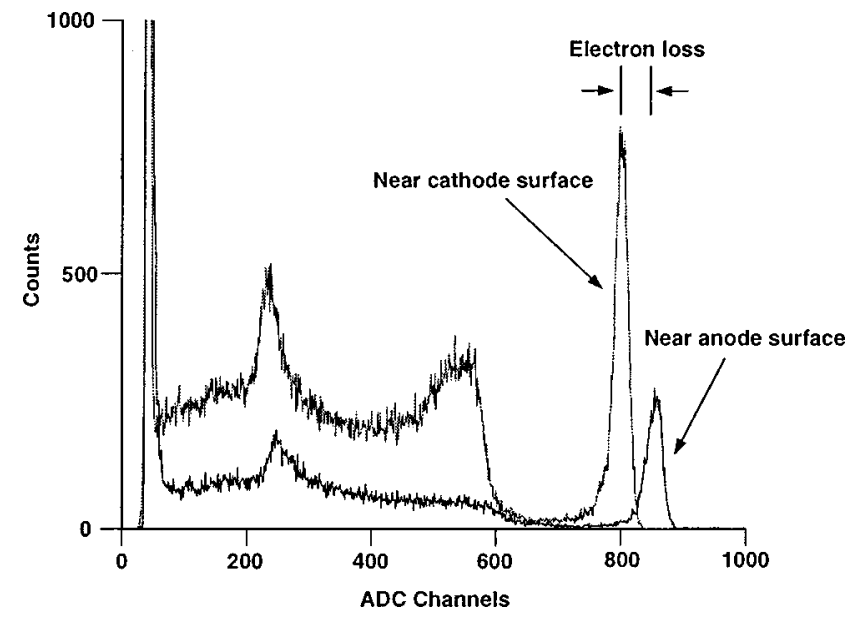

FIG. 1. Energy spectra of $662 \mathrm{keV} \gamma$ rays from interactions near the cathode and anode surfaces obtained from a $1 \mathrm{~cm}$ cube CdZnTe detector.

$$
\frac{d N}{d z}=-\frac{N}{\lambda_{e}},
$$

the number of electrons at drift length $z$ can be expressed as:

$$
N(z)=N_{0} \exp \left(-\frac{z}{\mu_{e} \tau_{e} E}\right) .
$$

By employing the single polarity charge sensing technique ${ }^{1}$ using an equal gain for the collecting and noncollecting anode signals, the pulse amplitude obtained from the coplanar grid anodes is proportional to the number of electrons collected at the anode surface. This behavior is in marked contrast with conventional induced signals in which the contribution of each electron is weighted by its drift distance. By using the depth sensing method, ${ }^{4,5}$ the electron drift length can be obtained independently. Since the number of electrons versus the electron drift length can be measured, the electron mean free drift length $\lambda_{e}$ or $\mu_{e} \tau_{e}$ can be directly obtained from the measurement. For example, Fig. 1 shows two energy spectra of $662 \mathrm{keV}\left({ }^{137} \mathrm{Cs}\right) \gamma$ rays, one restricted to interactions near the cathode surface and the other near the anode surface. Since the electron losses can be ignored when $\gamma$ rays deposit their energies near the anode surface, the difference of photopeak amplitudes between the two spectra directly shows the number of electrons that are lost when drifting from the cathode surface to the anode surface.

On a coplanar grid single polarity charge sensing device, when the relative gain between the collecting and noncollecting anodes is set to be 1.0 , the pulse rise time is determined by the electron drift time only through a distance of $\Delta z$ in the vicinity of the anode surface. $\Delta z$ is approximately the pitch of the coplanar grid anodes, generally much smaller than the detector thickness. Since the pulse rise time of such a device is much faster than that of a conventional planar detector, the ballistic deficit due to the variation of pulse rise time can be ignored on a coplanar grid CdZnTe detector if the shaping time constant is selected at a few $\mu$ s. Therefore, we can assume that the measured photopeak amplitudes are proportional to the number of electrons collected at the anode surface. In Eq. (3), let $N_{0}$ be the photopeak amplitude obtained from the anode surface, and $N(D)$ be the photopeak amplitude from events originating near the cathode surface, $\mu_{e} \tau_{e}$ can be obtained directly from the measurement data:

$$
\mu_{e} \tau_{e}=-\frac{D^{2}}{V \cdot \ln \left(N / N_{0}\right)},
$$

where $V$ is the bias voltage between the cathode and the anode.

There are two factors that could affect the precision of these measurements. The first is rooted in the assumption that the pulse amplitude is proportional to the number of electrons collected at the anode surface. This is only true when the relative gain between the collecting and noncollecting anodes is exactly 1.0. Otherwise, it effectively multiplies the total induced charge $Q$ by different constants when the $\gamma$-ray interaction depth varies. This depth dependent gain will distort the true loss of electrons. In practice, this means that not only does the relative gain of the subtraction have to be calibrated, but also the relative gain between the two preamplifiers. One method of calibration is to switch the polarity of bias voltage between the collecting and noncollecting anodes and to see whether the pulse amplitudes, which now have the opposite polarity, have the same amplitude after pulse shaping. This procedure involves changing both the bias voltage polarity between the two coplanar anodes, as well as the input polarity of the shaping amplifier. Second, there is a nonlinear region in the vicinity of the anode surface which could give a systematic error in the pulse amplitude for events that originate in this region. ${ }^{5}$ Therefore, there may be some systematic error introduced by assuming that the measured photopeak amplitude for events interacting near the anode is an accurate measure of $N_{0}$. This possible bias is intrinsic to the procedure and is not easily removed. With these caveats in mind, this technique can measure the electron $\mu_{e} \tau_{e}$ directly. In contrast to the conventional methods, it is not sensitive to the ballistic deficit, no curve fitting is involved, it is not affected by surface trapping, and it allows the use of common higher energy $\gamma$ rays.

\section{DIRECT MEASUREMENT OF $\mu_{e} \tau_{e}$ BY MEASURING PHOTOPEAK AMPLITUDES VERSUS $\lambda_{e}$}

While the technique discussed in Sec. II can provide an estimate of $\mu_{e} \tau_{e}$, a more accurate measurement procedure is possible which reduces the potential systematic errors from the calibration of the relative gain between the coplanar anodes and the effect due to the nonlinear region near the anode surface. An alternative technique of measuring $\mu_{e} \tau_{e}$ can be described as follows: measure the photopeak amplitudes $N_{1}$ and $N_{2}$ from events originating near the cathode surface at bias voltages $V_{1}$ and $V_{2}$ between the cathode and the anode, respectively. The photopeak amplitudes are proportional to the number of electrons collected at the anode surface in two cases. From Eq. (3):

$$
\begin{aligned}
& N_{1}=N_{0} \exp \left(-\frac{D^{2}}{\mu_{e} \tau_{e} V_{1}}\right), \\
& N_{2}=N_{0} \exp \left(-\frac{D^{2}}{\mu_{e} \tau_{e} V_{2}}\right) .
\end{aligned}
$$




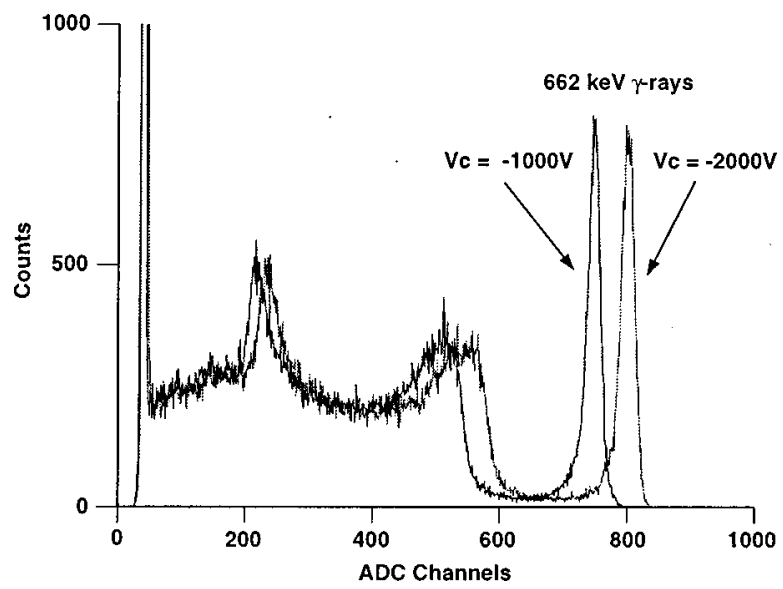

FIG. 2. Energy spectra of $662 \mathrm{keV} \gamma$ rays obtained from near the cathode surface at two bias voltages $V_{c}$. The detector is a $1 \mathrm{~cm}$ cube CdZnTe.

Solving yields:

$$
\mu_{e} \tau_{e}=\frac{D^{2}}{\ln \left(N_{1} / N_{2}\right)}\left(\frac{1}{V_{2}}-\frac{1}{V_{1}}\right)
$$

The original number of electron-hole pairs $N_{0}$ is canceled out.

An example of corresponding measurement data is shown in Fig. 2. The lower photopeak amplitude at lower bias voltage reflects the loss of electrons across the same detector thickness due to the shorter electron mean free drift length. Although the relative gain between the collecting and noncollecting anode may not be exactly 1.0 , since $\gamma$ rays originate from the cathode surface in both measurements, the depth dependent relative gains applied are nearly the same and are canceled out in $N_{1} / N_{2}$. Therefore, the possible systematic error caused by any deviation of relative gain between coplanar anodes from unity can be eliminated. In addition, since both spectra are collected from the cathode side, the possible error from the nonlinear region near the anode surface is greatly reduced. Since this technique is a relative measurement based on photopeak amplitude, and the only parameter changed during the two measurements is the bias voltage between the cathode and the anode, most of the potential sources of systematic error are eliminated. These include the change of detector performance versus $\gamma$-ray interaction depth, possible nonuniformity charge collection, etc. Because of the short rise time of the pulses from the coplanar electrodes, ballistic deficit is no longer an important issue.

When detector thickness $D$ and the bias voltages $V_{1}$ and $V_{2}$ are accurately known, the error in the measurement of $\mu_{e} \tau_{e}$ can be estimated by applying error propagation to Eq. (7):

$$
\left(\frac{\sigma\left(\mu_{e} \tau_{e}\right)}{\mu_{e} \tau_{e}}\right)^{2}=\frac{1}{\left[\ln \left(N_{1} / N_{2}\right)\right]^{2}}\left[\left(\frac{\sigma\left(N_{1}\right)}{N_{1}}\right)^{2}+\left(\frac{\sigma\left(N_{2}\right)}{N_{2}}\right)^{2}\right] .
$$

For simplicity, we can assume the distribution of photopeak amplitudes follows a Gaussian distribution with an expectation value of $N$, which is the centroid of the photopeak, and standard deviation $\sigma(H)$. The value of $\sigma(H)$ can be obtained from the full width half maximum (FWHM) of the pulse height spectrum as:

$$
\sigma(H) \approx \mathrm{FHWM} / 2.35
$$

and the standard deviation of the centroid $\sigma(N)$ can be obtained from $\sigma(H)$ and the total number of $\gamma$-ray events $M$ contributing to the photopeak:

$$
\sigma(N)=\frac{\sigma(H)}{\sqrt{M}} .
$$

With a typical measured energy resolution at $662 \mathrm{keV} \gamma$-ray energy of $2 \%$ FWHM, an electron transport loss fraction of $5 \%\left(N_{1} / N_{2}=0.95\right)$ and 10000 total counts under the photopeak, we can estimate the relative standard deviation of the measured $\mu_{e} \tau_{e}$ using Eqs. (8), (9), and (10):

$$
\frac{\sigma\left(\mu_{e} \tau_{e}\right)}{\mu_{e} \tau_{e}} \approx \frac{1}{|\ln 0.95|} \times \frac{2 \%}{2.35 \times \sqrt{10000}} \sqrt{2} \approx 0.23 \% .
$$

This estimate shows that the possible error caused by the measurement precision of $N_{1}$ and $N_{2}$ can be very small. In practice, the actual error may be determined by the limited depth resolution of the technique. At a $\gamma$-ray energy of 662 $\mathrm{keV}$, the typical depth resolution is a few percent of the detector thickness. ${ }^{5}$ This means that the electron drift length $z$, which was simplified as $D$ in Eq. (7), should be treated as a source of error while $N_{1}, N_{2}, V_{1}$, and $V_{2}$ are treated as constants in our error estimation. We can write Eq. (7) as:

$$
\mu_{e} \tau_{e}=\frac{z}{\ln \left(N_{1} / N_{2}\right)}\left(\frac{D}{V_{2}}-\frac{D}{V_{1}}\right) .
$$

This gives:

$$
\frac{\sigma\left(\mu_{e} \tau_{e}\right)}{\mu_{e} \tau_{e}}=\frac{\sigma(z)}{z} .
$$

In our measurements, we grouped $\gamma$-ray interaction depths into 20 bins, so $\sigma(z) / z \approx 5 \%$ and $\sigma\left(\mu_{e} \tau_{e}\right) /\left(\mu_{e} \tau_{e}\right) \approx 5 \%$.

\section{MEASUREMENT RESULTS}

Four CdZnTe detectors have been fabricated into coplanar grid single polarity charge sensing devices and their $\mu_{e} \tau_{e}$ values were measured. Since we developed the technique described in Sec. II prior to that described in Sec. III, our earlier measurements were based on Eq. (4). We believe that the method described in Sec. III is more accurate, so values of $\mu_{e} \tau_{e}$ were measured again using Eq. (7). Measurement results from both techniques are summarized in Table I. Although the measured $\mu_{e} \tau_{e}$ values are consistent from the two measurement techniques, as discussed in previous sections, the results obtained using Eq. (7) are believed more accurate. The crystals were obtained from eV Products. ${ }^{6}$ It is interesting that the $\mu_{e} \tau_{e}$ values are now smaller than the method of using Eq. (4). The probable reason for this is that we had selected the events beginning some distance (depth parameter was typically $3 / 20$ ) from the anode surface, instead of selecting those originating very close to the anode. As a result, the older measurements underestimated the electron loss 
TABLE I. Measured $\mu_{e} \tau_{e}$ values.

\begin{tabular}{lccc}
\hline \hline & \multicolumn{3}{c}{$\mu_{e} \tau_{e}\left(10^{-3} \mathrm{~cm}^{2} / \mathrm{V}\right)$} \\
\cline { 2 - 4 } \multicolumn{1}{c}{ Detector (ID) } & Using Eq. (4) & Using Eq. (7) & From Hecht relation $^{\mathrm{b}}$ \\
\hline $1 \times 1 \times 1 \mathrm{~cm}(704474)$ & 8.6 & 7.7 & N/A $^{\mathrm{c}}$ \\
$1.5 \times 1.5 \times 0.5 \mathrm{~cm}(\mathrm{~L} 1643 \# 1)$ & 8.6 & 7.2 & $\mathrm{~N}^{\mathrm{c}}$ \\
$1.5 \times 1.5 \times 1 \mathrm{~cm}(700033)$ & 5.9 & 4.1 & $2.5(\# \mathrm{C} 16-03)$ \\
$1 \times 1 \times 1 \mathrm{~cm}(1315-04)$ & 7.7 & 6.9 & $3.72(\# \mathrm{C} 17-04)$ \\
\hline \hline
\end{tabular}

${ }^{\mathrm{a}}$ Technique is more accurate.

${ }^{\mathrm{b}}$ Measured by eV Products.

${ }^{c}$ Not available.

which would have occured across the full depth of the detector. The choice was necessary because the detector performance had significant degradation for events originating very close to the anode surface on our early detectors.

\section{DISCUSSION}

It can be seen from Table I that our measured $\mu_{e} \tau_{e}$ values are significantly higher than those measured using a conventional method based on the Hecht relation. This difference may be generated by ballistic deficit and surface trapping which tend to produce a bias toward lower observed values of $\mu_{e} \tau_{e}$.

The new techniques, which directly measure electron $\mu_{e} \tau_{e}$ based on the combination of single polarity charge sensing and depth sensing methods, can significantly reduce the systematic error caused by ballistic deficit, allowing the use of high energy $\gamma$ rays which can penetrate deep inside the bulk of semiconductors. These new approaches can reduce possible error caused by different degrees of charge carrier trapping very close to the surface and within the bulk. These methods can take advantage of good signal-to-noise ratios from measurements using $\gamma$ rays at high energies. They do not require a pulsed laser, or the vacuum system associated with $\alpha$ source measurements. In particular, the technique described in Sec. III can provide more accurate results since it is a relative measurement. It should be noted that the new techniques can be used in conjunction with $\alpha$ sources and low energy $\gamma$ rays, in which case no depth sensing is necessary if radiations are incident from the cathode surface. These techniques can be used on other types of wide band-gap semiconductors, such as $\mathrm{HgI}_{2}$ and CdTe. Further- more, if a significant number of holes can traverse the semiconductor thickness, the new techniques could also be used to measure $\mu_{h} \tau_{h}$. The limitation of these techniques is that single polarity charge sensing must be employed.

Finally, since these techniques measure directly the number of electrons collected at the anode surface, the assumption of uniform charge trapping within the bulk of the semi-conductor is not mandatory. In the present measurements, we know that the measured depth parameter, which is the ratio of the cathode signal and the coplanar anode signal, is a monotonic function of the true depth of $\gamma$-ray interaction. ${ }^{4,5}$ The energy spectra from the events which originated from near the cathode and anode surfaces were obtained by selecting the depth parameter near its maximum and minimum values. If the measured depth parameter is calibrated in units of distance, the electron trapping at various depths of the detector could be measured.

\section{ACKNOWLEDGMENTS}

The authors would like to thank Dr. D. S. McGregor for valuable discussions. This work was supported under the U.S. Department of Energy, Grant No. DOE-FG0894NV11630.

${ }^{1}$ P. N. Luke, IEEE Trans. Nucl. Sci. 42, 207 (1995).

${ }^{2}$ Semiconductors for Room Temperature Nuclear Detector Applications, edited by T. E. Schlesinger and R. B. James, Semiconductors and Semimetals, Vol. 34 (Academic, New York, 1995), pp. 493-530.

${ }^{3}$ K. Hecht, Z. Phys. 77, 235 (1932).

${ }^{4}$ Z. He et al., Nucl. Instrum. Methods Phys. Res. A 380, 228 (1996).

${ }^{5}$ Z. He et al., Nucl. Instrum. Methods Phys. Res. A 388, 180 (1997).

${ }^{6} \mathrm{eV}$ Products, 375 Saxonburg Boulevard, Saxonburg, PA 16056. 